\title{
Assessing the efficacy of Canada's food guide and the barriers of use
}

\author{
Hana Dibe* \\ Department of Human Health and Nutritional Science, University of Guelph, Guelph, ON, Canada \\ *Author for correspondence (dibehana@gmail.com)
}

\begin{abstract}
:
The landscape of nutrition advice is vast and full of misinformation. A primary source of nutrition advice in Canada comes from the Canadian Food Guide, however, many questions remain regarding the reach and accessibility of the food guide. Specifically, is the population most likely to receive and use this information, the population that needs it the most? Are there barriers to following this guide that Health Canada has failed to address? Is there evidence supporting the efficacy of this food guide in populations at risk for nutrition misinformation or diet-related preventable diseases? This commentary reviews the past research regarding efficacy of previous food guides and highlights potential barriers preventing equal and accessible use of Canada's Food Guide.
\end{abstract}

\section{Dietary guidelines as a primary source of nutri- tion information}

The landscape of nutritional advice is vast and full of misinformation. For example, celebrity Gwyneth Paltrow's Netflix show "The Goop Lab" claims to provide scientifically backed advice on topics including fasting and veganism, but researchers and medical professionals have debunked many of their health claims [1]. Gwyneth's show is one among many other media outlets criticized for spreading misinformation, specifically in the area of nutritional advice $[2,3]$. Nutritional advice is abundant in internet blogs, lifestyle magazines, and from celebrities and self-claimed social media 'health experts,' many of whom do not have an equal standard of nutrition accreditation [2-4]. One study found that $54 \%$ of health information given out on social networking sites contained inaccurate content [5]. This spread of misinformation contributes to the increasing public confusion regarding nutritional eating choices, which can negatively impact perceptions or behaviours [6].

Globally, governments play a large role in regulating public health through nutritional recommendations in the form of food guides. Canada's Food Guides are created with expert input from dieticians, public health nurses, teachers, and physical activity specialists, and may help to counter the spread of misinformation by providing evidence-backed and fact-checked information [7]. Health Canada released the new, long-awaited Canada's Food Guide in March 2019, 12 years after the previous version was released in 2007. The new Food Guide adopts a plate-centric style, which includes sections on a plate of various food groups to represent qualitative intake proportions, and veers away from the past numeric formats where certain food groups were allotted a specific number of servings to have per day [8]. In addition to providing a variety of food options, the new Food Guide includes qualitative advice on how to eat across different life stages, and behavioural advice for healthy eating habits (i.e. eating with others). This supplementary information supports the idea that nutrition and healthy eating are not black and white concepts that require strict rules to follow.

\section{Efficacy and reach of Canada's food guide}

Canada's Food Guide is diligently backed up with reliable research, however, there is a lack of knowledge regarding the efficacy of its use or the use of government-backed nutritional guidelines in general. More specifically, is the population most likely to receive and use this information the population that needs it the most? Are there barriers to following this guide that Health Canada has failed to address? Is there evidence supporting the efficacy of this Food Guide in populations at risk for nutrition misinformation or diet-related preventable diseases such as diabetes? Although most of the research presented herein was conducted before the release of the current Food Guide, the reach and use of past Canadian Food Guides can be used to predict the efficacy of the current guide.

Data from the 2012 Canadian Community Health $(\mathrm{CCH})$ survey revealed that while most Canadians were aware of the Food Guide, less than one-third of respondents had a copy in their homes, of which most were women. Out
This is an Open Access article distributed under the terms of the Creative Commons Attribution License (CC BY 4.0)

License deed can be found at: http://creativecommons.org/licenses/ by $/ 4.0 /$

162 https://doi.org/10.29173/hsi291 Received 14 February 2020 Accepted 4 July 2020 
of the sources used for healthy eating information, 50\% of respondents selected "no sources," followed by 20\% for both "general research," "family and friends," and a mere $8.7 \%$ said they consult the Food Guide [9].

Preliminary data from a new survey $(n=1017)$ conducted by Dalhousie University and the University of Guelph revealed that $52 \%$ of Canadians face barriers to adopting the new Food Guide [10]. More than $25 \%$ of respondents cited that the new Food Guide would not be affordable to adopt, but calculations showed that on average, a family of four would save $6.8 \%$ more if they adopted the new Food Guide. However, with the push to increase fruit and vegetable consumption, the demand in produce over the next few years may increase which could in turn reduce the affordability of the Food Guide if fruit and vegetable prices were to spike [10].

Similar to the $2012 \mathrm{CCH}$ survey, participants placed the Food Guide $6^{\text {th }}$ for sources of healthy eating advice, behind family and friends, general research, social media, cookbooks and magazines, and TV programs, and less than $30 \%$ of Canadians had viewed the 2007 Food Guide in the past 12 months [10]. The lack of use in that year highlights the minimal impact the guide had on Canadian food choices. Detailed participant demographics were not revealed, except that they were above 18 years old and had lived in Canada for more than 12 months. Therefore, we cannot be confident the sample population represents the variety of ethnic and socioeconomic backgrounds present in Canada.

Research has shown that socioeconomic status (SES) is a predictor of diet quality $[11,12]$. SES is a measure of social status or class, and is often calculated as a combination of education, income, and occupation. With this is mind, it is speculative to state that lack of awareness, education, and use of national food guides is a potential contributor to low diet quality in those with a lower SES. Geographical barriers are also a large issue to people with low SES; in the United States, there are typically fewer chain grocery and more convenient stores in lower SES areas as well as in predominantly African American neighbourhoods in comparison to Caucasian and high SES ones [12]. There is also an intersection of low SES and racial minorities in certain geographic locations, and this may further contribute to the reduced chain-grocery store abundance in both these areas [13]. Poor access to supermarkets and an ensuing dominance of convenience stores with mainly energy dense and nutrient lacking foods is an inevitable set-up for poor adherence to nutritional guidelines [14]. If barriers are not removed to allow for healthy food choices, then a national food guide cannot confidently expect to impact the population that may benefit from these recommendations the most.

\section{Notable advancements and future directions}

Arguably, the government's simplified plate approach, with no quantitative food recommendations, is flexible and adaptable. Individuals can swap the marketed fresh fruits and vegetables with frozen counterparts, instead of using canned forms, which often contain excess sugar or salt. The Food Guide also contains over 30 diverse recipes, posted on the online Canada's Food Guide website, from a variety of cuisines that are in line with the recommendations. Perhaps these recipes could have alternate instructions on how to prepare using frozen, canned, or dried ingredients to increase accessibility for the variety of SES backgrounds.

Healthy eating is just as important for ones' mental health as it is for physical health, and arguably one's psychological attitude towards food (e.g. having anxiety towards eating) can impact their food choices [15]. There are associations linking a diet high in refined sugars with increased mental illness symptoms, further backing the need to address the psychological factor of healthy eating $[16,17]$. The emphasis on food behaviours including "eating with others" or "cook your own meals" is an acknowledgeable component of the new food guide and highlights the psychological component of healthy eating. A statement from the Community Food Centres Canada described this guidance as "a critical step forward is the inclusion of advice not only on what we eat but how we eat - cooking more at home, enjoying food, and eating with others - which, taken together, encourage a more communal and healthful approach to eating." [18]. These behavioural changes in eating habits may be more easily implemented irrespective of SES and cultural background.

The efforts of the government should not go unnoticed and the 2019 Food Guide is a move in the right direction. In order to maintain a level of inclusivity expected from such a governing body, we need evidence-based research concerning the use and accessibility for people of lower SES and various cultures. The past studies conducted on the use of the 2007 food guide should be repeated with the new food guide to assess whether the food guide itself or it's updated marketing tactics (i.e. advertisements on social media) can influence consumer use. Additionally, there needs to be organized programs in place to increase nutrition awareness and educate on reliable sources for nutrition information, as well as resources on how to use the guide on a budget. Future research should aim to address if the past or current Food Guide has been associated with beneficial health effects such as reducing type 2 diabetes incidence across Canada and if there is potential benefit of increased food guide marketing to at-risk populations. Canada's Food Guide has the potential to play an important role in the lives of Canadians but there is more work to be done to ensure optimal use and its role in countering the spread of nutrition misinformation.

\section{References}

1. Meghan Collie. Experts fact-check health claims in Netflix's 'The Goop Lab' [Internet]. Global News. 2020. Available from: https:// globalnews.ca/news/6440674/the-goop-lab-fact-check/

2. Horsburgh H, Barron D. Who are the experts? Examining the online promotion of misleading and harmful nutrition information. In: Medical Misinformation and Social Harm in Non-Science-Based Health Practices. Routledge; 2019.

3. Hoffman SJ, Mansoor Y, Natt N, Sritharan L, Belluz J, Caulfield T, et al. Celebrities' impact on health-related knowledge, attitudes, behaviors, and status outcomes: Protocol for a systematic review, meta-analysis, and meta-regression analysis. Syst Rev [Internet]. 2017;6(1):1-13. Available from: http://dx.doi.org/10.1186/s13643- 
016-0395-1

4. Wansink B. Food and nutrition misinformation. J Am Diet Assoc. 2006;106(4):601-7.

5. Tsai CC, Tsai SH, Zeng-Treitler Q, Liang BA. Patient-centered consumer health social network websites: a pilot study of quality of user-generated health information. AMIA Annu Symp Proc. 2007;16:1137.

6. Cornish L, Moraes C. The Impact of Consumer Confusion on Nutrition Literacy and Subsequent Dietary Behavior. Psychol Mark [Internet]. 2015;32(5):558-74. Available from: http://eprints.lancs. ac.uk/23431/

7. Caulfield T, Marcon AR, Murdoch B, Brown JM, Perrault ST, Jerry J, et al. Health Misinformation and the Power of Narrative Messaging in the Public Sphere. Can J Bioeth. 2019;2(2).

8. Canada's Food Guide. Health Canada. 2019.

9. Slater JJ, Mudryj AN. Are we really "eating well with Canada's food guide"? BMC Public Health. 2018;18(1):1-7.

10. Charlebois S, Wambui B, Racey M, Fiander D, Smook M, Somogyi $S$, et al. Canada's Food Guide: Awareness, Understanding, Affordability, and Barriers to Adoption (Preliminary Results). Dalhousie Univ Univ Guelph. 2019;1-25.

11. Darmon N, Drewnowski A. Does social class predict diet quality? Am J Clin Nutr. 2008;87(5):1107-17.

12. White R. Examining the Effect of Socioeconomic Status on Access to Nutritional Food. Perspect (University New Hampshire) [Internet]. 2012;Spring(March):114-20. Available from: https://cola.unh.edu/ sites/cola.unh.edu/files/student-journals/P12_White.pdf

13. Williams DR, Mohammed SA, Leavell J, Collins C. Race, Socioeconomic Status and Health: Complexities, Ongoing Challenges and Research Opportunities. Ann N Y Acad Sci. 2010;1186:612-5.

14. Jack D, Neckerman K, Schwartz-Soicher O, Lovasi GS, Quinn J, Richards C, et al. Socio-economic status, neighbourhood food environments and consumption of fruits and vegetables in New York City. Public Health Nutr. 2013;16(7):1197-205.

15. Webb C, Thuras P, Peterson C, Lampert J, Miller D, Crow S. Eating-related anxiety in individuals with eating disorders. Eat Weight Disord. 2011;16(4):e236-41.

16. Marx W, Moseley G, Berk M, Jacka F. Nutritional psychiatry: The present state of the evidence. Proc Nutr Soc. 2017;76(4):427-36.

17. Knüppel A, Shipley MJ, Llewellyn CH, Brunner EJ. Sugar intake from sweet food and beverages, common mental disorder and depression: Prospective findings from the Whitehall II study. Sci Rep. 2017;7(1):1-10.

18. Community Food Centres Canada. Community Food Centres Canada applauds the update to Canada's Food Guide. 2019.

(c) The Author(s) 